15. Hagenaar, H. J., Schrijver, C. J., Title, A. M. \& Shine, R. A. Dispersal of magnetic flux in the quiet solar photosphere. Astrophys. J. (submitted)

16. Schrijver, C. J., Coté, J., Zwaan, C. \& Saar, S. H. Relations between the photospheric magnetic field and the emission from the outer atmospheres of cool stars. I. The solar Ca II K line core emission. Astrophys. J. 337, 964-976 (1989).

17. Schrijver, C. J. in Stellar Surface Structure (eds Strassmeier, K. G. \& Linsky, J. L.) (IAU Symp. 176 Kluwer Academic, Dordrecht, 1996)

\section{The signature of chemical valence in the electrical conduction through a single-atom contact}

Elke Scheer $* \dagger$, Nicolás Agraïtł, Juan Carlos Cuevas $\$$, Alfredo Levy Yeyati§, Bas Ludoph\|, Alvaro Martín-Rodero\$, Gabino Rubio Bollinger $\ddagger$, Jan M. van Ruitenbeek\| \& Cristián Urbina*

* Service de Physique de l'Etat Condensé, CEA-Saclay, F-91191 Gif-sur-Yvette, France

\$Laboratorio de Bajas Temperaturas, Departamento de Física de la Materia Condensada C-III, Instituto Universitario de Ciencia de Materiales "Nicolás Cabrera", Universidad Autónoma de Madrid, E-28049 Madrid, Spain $\$$ Departamento de Física Teórica de la Materia Condensada C-V, Universidad Autónoma de Madrid, E-28049 Madrid, Spain

|| Kamerlingh Onnes Laboratorium, Leiden University, PO Box 9506, 2300 RA Leiden, The Netherlands

Fabrication of structures at the atomic scale is now possible using state-of-the-art techniques for manipulating individual atoms ${ }^{1}$, and it may become possible to design electrical circuits atom by atom. A prerequisite for successful design is a knowledge of the relationship between the macroscopic electrical characteristics of such circuits and the quantum properties of the individual atoms used as building blocks. As a first step, we show here that the chemical valence determines the conduction properties of the simplest imaginable circuit-a one-atom contact between two metallic banks. The extended quantum states that carry the current from one bank to the other necessarily proceed through the valence orbitals of the constriction atom. It thus seems reasonable to conjecture that the number of current-carrying modes (or 'channels') of a one-atom contact is determined by the number of available valence orbitals, and so should strongly differ for metallic elements in different series of the periodic table. We have tested this conjecture using scanning tunnelling microscopy and mechanically controllable break-junction techniques ${ }^{2,3}$ to obtain atomic-size constrictions for four different metallic elements $(\mathrm{Pb}, \mathrm{Al}, \mathrm{Nb}$ and $\mathrm{Au})$, covering a broad range of valences and orbital structures. Our results demonstrate unambiguously a direct link between valence orbitals and the number of conduction channels in one-atom contacts.

The electrical conductance of a quantum coherent structure accommodating $N$ channels is given by the Landauer formula $G=G_{0} \Sigma_{n=1}^{N} T_{n}$, where $G_{0}=2 e^{2} / h$ is the conductance quantum ( $e$ is the electron's charge and $h$ is Planck's constant $)^{4}$, and $T_{n}$ is the transmission probability of the $n$th channel. Measuring the conductance is equivalent to measuring the sum $\Sigma_{n=1}^{N} T_{n}$, and gives no information about the individual $T_{n} \mathrm{~s}$. A recently developed technique based on the nonlinearities in the current-voltage characteristics (referred to here as IVs) of superconducting constrictions provides the latter information ${ }^{5}$. These nonlinearities, called 'subgap structure ${ }^{6}$, are sensitive to the exact values of the individual transmissions and can therefore be used as a tool to determine the channel ensemble, $\left\{T_{n}\right\}$.

In the ground state of a superconductor, all electrons are paired and there is an energy gap $\Delta$ for the creation of a quasiparticle excitation, that is, an unpaired electron. When transferring an electron between two banks in their ground state, two quasiparticles are created, one on each bank, and the energy $2 \Delta$ has to be provided by the voltage source in the circuit. The consequence is that a gap occurs in the single-electron transport characteristic for voltages $V<2 \Delta / e$. In addition, multiple Andreev reflections ${ }^{6}$ are possible, involving the transfer of $m$ electrons while two quasiparticles are created. These processes, which have a probability proportional to $\left(T_{n}\right)^{m}$ for small $T_{n}$, generate current steps at voltages $V=2 \Delta / m e$ within the gap. This is the origin of the sub-gap structure, the exact shape of which depends on $\left\{T_{n}\right\}$. Several authors ${ }^{7-9}$ have calculated the current voltage characteristic $i(T, V)$ of a single superconducting channel with arbitrary $T$.

We have used various experimental methods to achieve stable atomic-size contacts: a scanning tunnelling microscope (STM) for the measurements on $\mathrm{Pb}$ (ref. 10), mechanically controllable break junctions for $\mathrm{Nb}$ (ref. 2), and lithographically fabricated versions of the latter (ref. 3) for $\mathrm{Al}$ and Au. Figure 1 shows the typical development of the conductance $G$ for the four metals as the contacts are stretched. The total conductance decreases by steps until the contact breaks. After that it decreases exponentially with distance, the signature of the tunnel regime. The step heights are in the order of $G_{0}$, while the series of "plateaux" is different for each individual opening of the contact. It has been shown that the origin of the steps is in atomic rearrangements ${ }^{11-14}$ which occur while the constriction is elongated. The typical value of $G$ at which the contact breaks, the lengths of the plateaux, and the behaviour within the plateaux are characteristic of each material. $\mathrm{Pb}$ shows plateaux with negative slope, and an almost continuous decrease of $G$ between $3 G_{0}$ and $1 G_{0}$, where the contact usually breaks. Al has plateaux with positive slope, and breaks at around $G_{0}$ with a jump to $G<0.1 G_{0}$. $\mathrm{Nb}$ displays negatively inclined plateaux, smallest contacts with conductances between $2 G_{0}$ and $3 G_{0}$, and a jump to a tunnelling conductance as high as $G \approx G_{0}$. For our lithographic Au samples we obtain less well defined plateaux. The last contact before break typically has a conductance between $0.3 G_{0}$ and $0.8 G_{0}$.

We can stop the elongation at any point and record the IVs. With the junction in the vacuum tunnel regime, the IV corresponds well to the one expected for a single channel as shown for $\mathrm{Pb}$ in curve e of Fig. 2. In the contact regime one observes IVs as depicted in curves a to $\mathrm{d}$ of the same figure. These curves were recorded on the same sample for different last plateaux before breaking. It is usually assumed that these smallest stable contacts are one-atom contacts ${ }^{2}$. Our present analysis will give further support to this assumption. The occurrence of IVs with different sub-gap structure for the same total conductance (see Fig. 2) reveals the importance of the geometrical environment of the central atom for the conduction properties. In general, the IVs in the contact regime cannot be described by the single channel theory. We shall assume that the total current can be decomposed as:

$$
I(V)=\sum_{n=1}^{N} i\left(T_{n}, V\right)
$$

where $i\left(T_{n}, V\right)$ is the current of channel $n$. (This approximation is justified as the multiple Andreev processes do not mix the normal conduction channels, see, for instance, ref. 15.) The solid curves in Fig. 2 are fits to the experimental IVs using equation (1), and the theoretical curves $i(T, V)$ calculated in ref. 8, with the set $\left\{T_{n}\right\}$ and the number of channels $N$ as fitting parameters. Details of the analysis are given in ref. 5 .

For $\mathrm{Pb}$ it turns out that three or four channels contribute to the total current for the smallest contacts. Despite the fact that more 
than two channels are available, $G$ can be well below $2 G_{0}$. For larger contacts with $G \geqslant 3 G_{0}$, we find six or more channels. The numbers $N$ of channels we found at the different plateaux are indicated in Fig. 1. Only channels with a transmission larger than $1 \%$ of the total transmission are taken into account when determining $N$. Applying a similar analysis to different metals, we arrive at the important conclusion that the maximum number of channels $N_{\max }$ for the smallest contacts is characteristic for a given metal. In the IVs of $\mathrm{Al}$

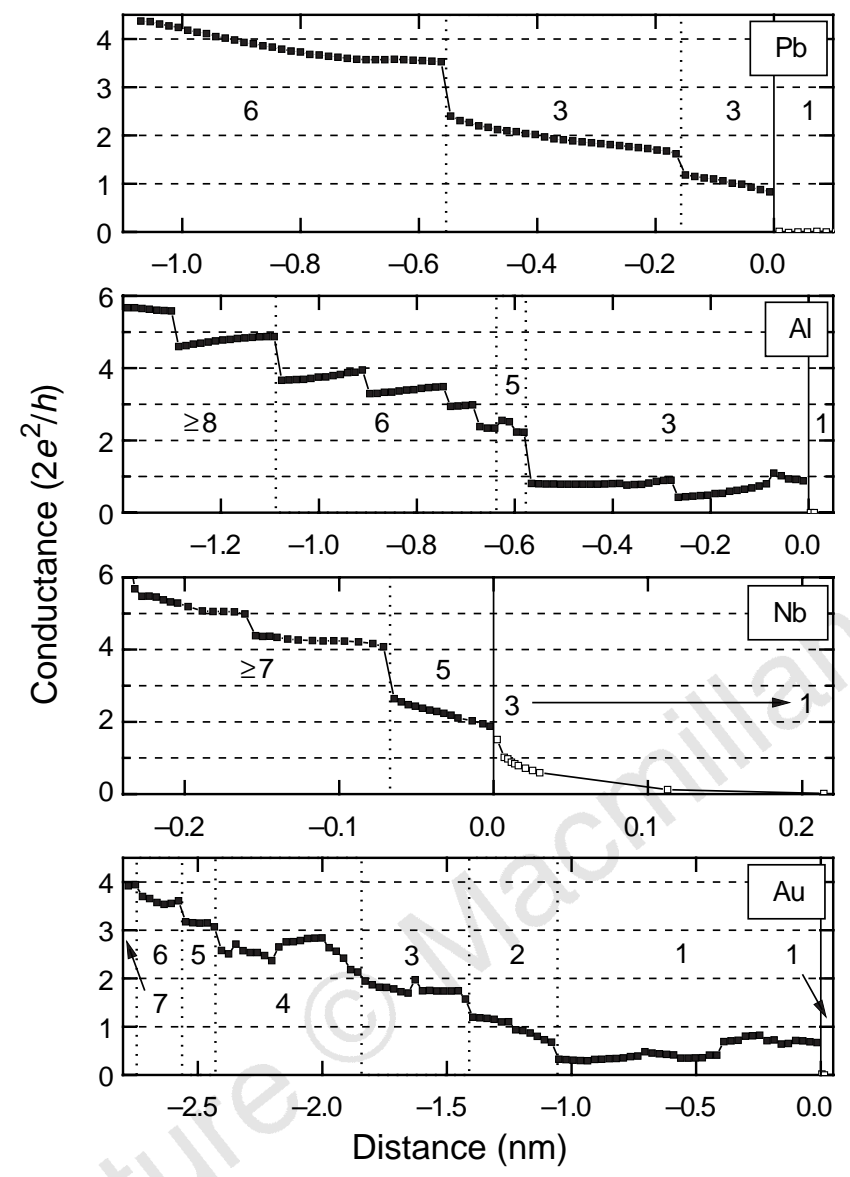

Figure 1 Typical conductance $G$ as a function of distance, recorded during a continuous opening of the samples, for four different metals. The distribution of transmission values $\left\{T_{n}\right\}$ is established by fitting the IVs recorded at each point. The $\mathrm{Pb}$ data have been taken with a STM at $1.5 \mathrm{~K}$. For preparing few-atom contacts with the STM, the $\mathrm{Pb}$ tip is indented repeatedly into the $\mathrm{Pb}$ sample surface and finally withdrawn ${ }^{10}$. For $\mathrm{Al}$ and $\mathrm{Au}$ we used lithographic break junctions below $100 \mathrm{mK}$. The initial samples before breaking are suspended nanobridges made of evaporated thin films with a central constrictions of about $200 \mathrm{~nm} \times 200 \mathrm{~nm}$ (ref. 3). The Nb data have been measured on a classical break junction, that is, a notched wire glued on top of a flexible substrate ${ }^{2}$, at $1.6 \mathrm{~K}$. By bending the substrate the break junctions are elongated and finally broken. The opening speeds were in all cases in the range $1-10 \mathrm{pm} \mathrm{s}^{-1}$. The numbers indicate the number $N$ of conduction channels in each region. The symbol " $\geq$ " means that only a lower bound for $N$ could be determined, because of systematic deviations from the theory due to a modified quasiparticle density of states (Nb) or because of experimental scattering (Al) which limits the maximum $N$ that can be disentangled. Plateaux corresponding to different $N$ are separated by dotted vertical lines. The horizontal arrow indicates that $N$ decreases in the tunnel regime. Filled symbols indicate contact regime, open symbols the tunnel regime. The origins of the distance axes are arbitrarily set at the points where the contacts break. The distance scales for $\mathrm{Al}, \mathrm{Nb}$, and $\mathrm{Au}$ have been extracted from the exponential distance dependence of the current in the tunnel regime. The distance scale for the measurements on $\mathrm{Pb}$ has been determined using the calibration of the STM and is consistent with that deduced from the tunnel regime. smallest contacts we find typically the contributions of three channels, whereas for $\mathrm{Nb}$ we detect five channels. These results support our initial hypothesis that $N_{\max }$ for one-atom contacts is limited by the number of valence orbitals of the central atom $N_{\text {orb}}$.

To gain insight into how the conduction channels are actually built up from the atomic orbital structure we have constructed a model of a one-atom constriction using an atomic orbital basis ${ }^{16,17}$. In references 16 and 17 it is explained in detail how the theoretical $\left\{T_{n}\right\}$ can be obtained, and how the different atomic orbitals at the central atom contribute to each conduction channel as a function of the atomic arrangement of the contact. Of course, the detailed atomic positions of the atoms in the experiments are unknown and quantitative comparison with theory is difficult. However, it turns out that the basic predictions of the model, in particular the number $N$ of conduction channels, are independent of the exact geometry chosen for the calculation. Figure 3 illustrates schematically the main results of our theoretical analysis for a one-atom contact between two closed packed pyramids (Fig. 3a) for $\mathrm{Al}$ (left) and $\mathrm{Pb}$ (right), for which one $s$ orbital and three $p$ orbitals have to be considered: due to the coupling of the central atom with its neighbours, the isolated atom's energy levels (thick vertical bars in Fig. $3 \mathrm{~b}$ and c) broaden to form a continuous local density of states (LDOS) in the neck (Fig. $3 \mathrm{~d}$ and e). The central atom has less neighbours than an atom in the bulk material, and therefore the bands are narrower than deep inside the perfect crystal (Fig. 3b and c). In the neck geometry, the central-atom $p_{z}$ orbital $(z$ is the transport direction) splits off from the $p_{x}$ and $p_{y}$ orbitals and hybridizes with the $s$ orbital, building a channel of mixed $s p_{z}$ character. The actual transport properties of the atomic contact are determined by the $T_{n}$ values at the Fermi level $E_{\mathrm{F}}$ which we determined self-consistently by imposing local charge neutrality (Fig. $3 \mathrm{f}$ and $\mathrm{g})^{16,17}$.

For the perfectly ordered $\mathrm{Pb}$ contact the main predictions are: (1) three channels have a significant $T_{n}$ around the Fermi energy, the fourth one having $T_{n}<10^{-3}$ (not visible in Fig. 3); (2) the total transmission is close to 2.5 around $E_{\mathrm{F}}$ (3) there is one mode which is widely open for a broad energy range, and (4) the second transmission eigenvalue is two-fold degenerate and has a smaller value

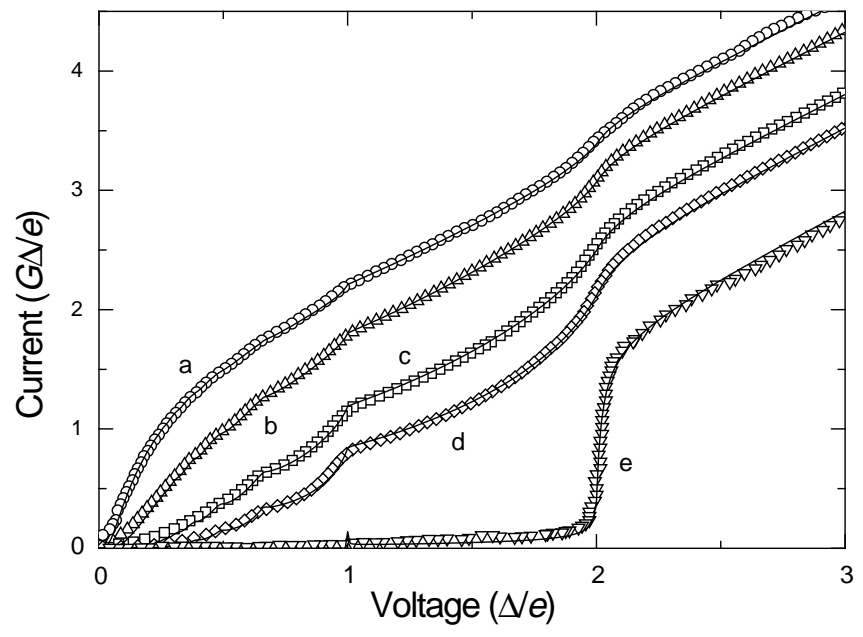

Figure 2 Measured current-voltage characteristics (plotting symbols) of five different configurations of a Pb sample at $1.5 \mathrm{~K}$ using STM, and best numerical fits (lines). Curves a-d have a very similar total transmission, close to 1.4; curve e has been taken in the tunnel regime. The individual channel transmissions obtained from the fits are: curve a, $T_{1}=0.955, T_{2}=0.355, T_{3}=0.085, T_{4}=0.005$; curve $b$, $T_{1}=0.89, \quad T_{2}=0.36, \quad T_{3}=0.145, \quad T_{4}=0.005 ;$ curve $c, T_{1}=0.76, T_{2}=0.34$, $T_{3}=0.27, T_{4}=0.02$; curve d, $T_{1}=0.65, T_{2}=0.34, T_{3}=0.29, T_{4}=0.12$; curve e, $T=0.026$. Voltage and current are in reduced units. The measured superconducting gap was $\Delta / e=1.37 \mathrm{mV}$. 
around $E_{\mathrm{F}}$ The widely open channel can be identified with the symmetric combination of $s$ and $p_{z}$. The two degenerate modes mainly correspond to the $p$ orbitals perpendicular to the $z$ direction. The degeneracy of the $p_{x}, p_{y}$ modes is due to the symmetry of the perfectly ordered model geometry and is lifted when disorder is introduced. Furthermore, disorder slightly reduces the $T_{n}$ of all channels without altering the gross features ${ }^{16}$.

These predictions are in good agreement with our experimental observations: smallest $\mathrm{Pb}$ contacts just before breaking most frequently have a $G$ between $1 G_{0}$ and $3 G_{0}$. The decomposition usually results in three or four channels, where the $T_{n}$ of the fourth one, if present, is usually smaller than 0.03 . We do not find last plateaux before breaking with only one or two, or with five, channels. Typically the analysis of the last plateau yields one well transmitted mode with $T_{n}>0.6$ and two smaller non-degenerate ones with $T_{n} \leqslant 0.4$. We attribute the lack of degeneracy and the smaller $T_{n} \mathrm{~s}$ to an asymmetrical environment around the central atom in the experimental contacts.

The case of $\mathrm{Al}$ is qualitatively similar because the relevant orbitals are of the same nature. However, the energy difference between the atomic $s$ and $p$ levels is smaller than in $\mathrm{Pb}$. Furthermore, there are only three valence electrons and therefore $E_{\mathrm{F}}$ is lower. Our model predicts again three open channels for a perfect neck-shaped oneatom contact with a total conductance close to $G_{0}$. Experimentally,
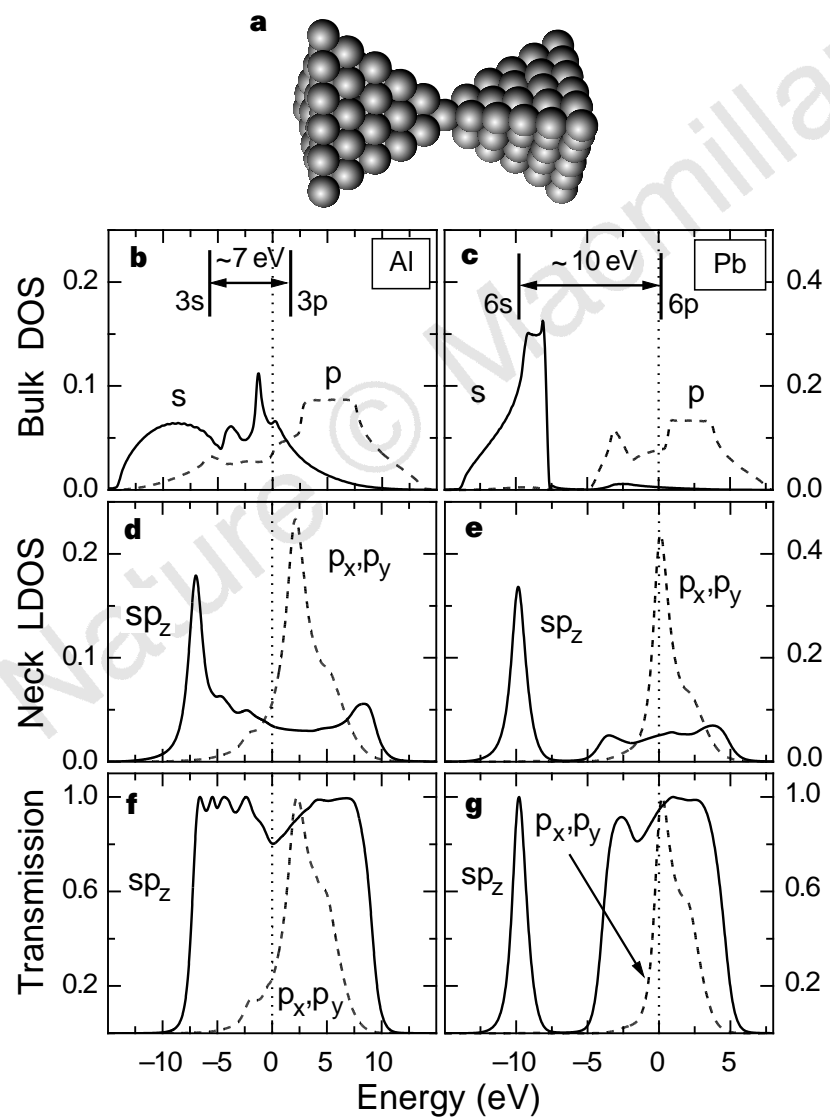

Figure $\mathbf{3}$ Localized orbitals model for electrical conduction through one-atom contacts. a, Model geometry. $\mathbf{b}-\mathbf{g}$, Schematic development of the atomic valence levels (thick vertical bars in $\mathbf{b}$ and $\mathbf{c}$ ) into bulk conduction bands (b and $\mathbf{c}$ ) for $\mathrm{Al}$ and $\mathrm{Pb}$. The panels $\mathbf{d}$ and $\mathbf{e}$ depict schematically the local density of states (LDOS) in $\mathrm{eV}^{-1}$ at the central atom of the model geometry. The global energy dependence of the transmission coefficients $T_{n}$ is shown in $\mathbf{f}$ and $\mathbf{g}$. The dotted lines indicate the position of the Fermi level. The $s p_{z}$ mode is the best transmitted mode for both materials. The $p_{x}$ and $p_{y}$ modes are degenerate due to the symmetry of the model geometry. The indicated energy differences between the atomic $s$ and $p$ levels are those used to fit the bulk band structure. we obtain a last plateau with $G_{0} \approx G_{0}$, decomposable into three channels. The case of an $\mathrm{Al}$ atom sandwiched between two flat surfaces has already been treated with density functional ${ }^{18}$ and other $a b$ initio calculations ${ }^{19}$, which predicted $G \approx 2 G_{0}$.

For $\mathrm{Nb}$ one-atom contacts, $N_{\max }=6$ as the density of states around $E_{\mathrm{F}}$ is mainly determined by the contributions of one $5 s$ and five $4 d$ orbitals. The model calculations for $\mathrm{Nb}$ yield five channels with non-vanishing $T_{n}$ (ref. 16) adding up to $G \approx 2.8 G_{0}$, again in agreement with the experimental findings (see third panel of Fig. 1). The experimental determination of $\left\{T_{n}\right\}$ is less precise than for $\mathrm{Pb}$ and $\mathrm{Al}$, because the quasiparticle density of states for $\mathrm{Nb}$ differs slightly from the one used for calculating the $i(T, V)$. (Deviations of the quasiparticle density of states from the case considered in the theory mostly affect the shape of the current steps around $V=2 \Delta / m e$ but not the current values in between. Therefore the analysis with the unmodified density of states is still possible.) An additional difference from the $\mathrm{Pb}$ and $\mathrm{Al}$ case is observed in the tunnel regime: for $\mathrm{Nb}$ contacts just after breaking, three channels contribute to the tunnel current. With increasing distance between the electrodes, one channel becomes dominating.

The cases discussed so far correspond to superconducting elements having several valence orbitals per atom. A crucial test for the validity of our analysis would be provided by monovalent metals like $\mathrm{Au}$ and $\mathrm{Na}$. They can be described by a single $s$ orbital, and the model predicts a single channel with $T \approx 1$ for a one-atom contact $^{16,17}$ in accordance with calculations based on molecular dynamics simulations ${ }^{20}$. As these monovalent metals are not superconducting, our method of characterizing channels is not directly applicable. However, a small piece of normal metal in good contact with a large piece of a superconductor develops a gap in its quasiparticle density of states ${ }^{21}$. The lithographic mechanically controllable break junction technique ${ }^{3}$ allows for the fabrication of a constriction where the central region consists of $\mathrm{Au}$ atoms, embedded in a superconducting $\mathrm{Al}$ environment. Using shadow evaporation through a suspended mask we evaporated 400-nmthick $\mathrm{Al}$ electrodes separated by a 50 -nm-wide gap, which is filled with a 20 -nm-thick Au layer deposited at a different angle under the same vacuum. The large asymmetry in thickness limits the influence of the normal metal on the quasiparticle density of states. As depicted in the lowermost panel of Fig. 1, we find indeed that the smallest Au contacts accommodate one single channel, contrary to the results for the $s p$-like and transition metals. However, we usually measure transmission values significantly below one. As for the other metals we interpret this reduced $T$ with respect to the model predictions as arising from disorder in the contact. Our Au samples are presumably more prone to disorder because of the very small $\mathrm{Au}$ layer thickness. Considering that the accuracy in determining $\left\{T_{n}\right\}$ is also limited because of the modified quasiparticle density of states, the single channel fit is still very satisfactory in the case of Au.

We draw two conclusions from our results. First, they provide clear evidence that the smallest contacts produced by the different experimental techniques are indeed one-atom contacts, as $N$ never exceeds $N_{\text {orb }}$. We stress that in these experiments a single atom determines a macroscopic quantity, that is, the total conductance of the macroscopic circuit in which it is embedded. The current which an one-atom contact can sustain can be as large as $100 \mu \mathrm{A}$, corresponding to the very large current density of $10^{11} \mathrm{~A} \mathrm{~cm}^{-2}$. This large current is carried by a small number of conduction channels. Second, the ensemble of our experimental results shows unambiguously that the conduction channels in an atomic contact are determined by the chemical nature of the central atom. As a crude rule of thumb, the number $N$ of active channels corresponds to the number of valence electrons. This essential fact cannot be understood within a free electron model. Only a microscopic model that takes into account the atomic orbital structure as well as the local atomic geometry can fully explain the conduction properties of these contacts. We expect that these concepts will be applicable, in 
slightly modified version, to conduction through clusters of atoms and molecules.

Received 29 December 1997; accepted 22 April 1998

1. Crommie, M. F., Lutz, C. P. \& Eigler, D. M. Confinement of electrons to quantum corrals on a metal surface. Science 262, 218-220 (1993).

2. van Ruitenbeek, J. M. in Mesoscopic Electron Transport (eds Sohn, L. L., Kouwenhoven, L. P. \& Schön, G.) 549-579 (Kluwer Academic, Dordrecht, 1997).

3. van Ruitenbeek, J. M. et al. Adjustable nanofabricated atomic size contacts. Rev. Sci. Instrum. 67, 108 111 (1996).

4. Landauer, R. Electrical resistance of disordered one-dimensional lattices. Phil. Mag. 21, 863-867 (1970).

5. Scheer, E., Joyez, P., Esteve, D., Urbina, C. \& Devoret, M. H. Conduction channel transmissions of atomic-size aluminum contacts. Phys. Rev. Lett. 78, 3535-3538 (1997).

6. Octavio, M., Tinkham, M., Blonder, G. E. \& Klapwijk, T. M. Subharmonic energy-gap structure in superconducting constrictions. Phys. Rev. B 27, 6739-6746 (1983).

7. Averin, D. \& Bardas, A. AC Josephson effect in a single quantum channel. Phys. Rev. Lett. 75, 18311834 (1995).

8. Cuevas, J. C., Martín-Rodero, A. \& Levy Yeyati, A. Hamiltonian approach to the transport propertie of superconducting quantum point contacts. Phys. Rev. B 54, 7366-7379 (1996).

9. Bratus, E. N., Shumeiko, V. S., Bezuglyi, E. V. \& Wendin, G. dc-current transport and ac Josephson effect in quantum junctions at low voltage. Phys. Rev. B 55, 12666-12677 (1997).

10. Agrait, N., Rodrigo, J. G. \& Vieira, S. Conductance steps and quantization in atomic-size contacts Phys. Rev. B 47, 12345-12348 (1996).

11. Rubio, G., Agraït, N. \& Vieira, S. Atomic-sized metallic contacts: mechanical properties and electronic transport. Phys. Rev. Lett. 76, 2302-2305 (1996).

12. Krans, J. M., van Ruitenbeek, J. M., Fisun, V. V., Yanson, I. K. \& de Jongh, L. J. The signature of conductance quantization in metallic point contacts. Nature 375, 767-769 (1995)

13. Landman, U., Luedtke, W. D., Salisbury, B. E. \& Whetten, R. L. Reversible manipulations of room temperature mechanical and quantum transport properties in nanowire junctions. Phys. Rev. Lett. 77, $1362-1365$ (1996).

14. Todorov, T. N. \& Sutton, A. P. Jumps in electronic conductance due to mechanical instabilities. Phys Rev. Lett. 70, 2138-2141 (1993).

15. Beenakker, C. W. J. Quantum transport in semiconductor superconductor microjunctions. Phys. Rev. B 46, 12841-12844 (1992).

16. Cuevas, J. C., Levy Yeyati, A. \& Martín-Rodero, A. Microscopic origin of the conducting channels in metallic atomic-size contacts. Phys. Rev. Lett. 80, 1066-1069 (1998).

17. Levy Yeyati, A., Martín-Rodero, A. \& Flores, F. Conductance quantization and electron resonances in sharp tips and atomic size contacts. Phys. Rev. B 56, 10369-10372 (1997).

18. Lang, N. D. Resistance of atomic wires. Phys. Rev. B 52, 5335-5342 (1995).

19. Wan, C. C., Mozos, J.-L., Taraschi, G., Wang, J. \& Guo, H. Quantum transport through atomic wires. Appl. Phys. Lett. 71, 419-421 (1997).

20. Brandbyge, M., Sørensen, M. R. \& Jacobsen, K. W. Conductance eigenchannels in nanocontacts. Phys. Rev. B 56, 14956-14959 (1997).

21. Belzig, W., Bruder, C. \& Schön, G. Local density of states in a dirty normal metal connected to a superconductor. Phys. Rev. B 54, 9443-9448 (1996).

Acknowledgements. We thank D. Esteve and M. H. Devoret for discussions and C. Sürgers for graphics preparation; N.A. and G.R.B. thank S. Vieira for discussions and support. The work was supported in part by the Deutsche Forschungsgemeinschaft (DFG), the Spanish CICYT, "Stichting FOM" (NWO) and the Bureau National de Métrologie (BNM)

Correspondence and requests for materials should be addressed to E.S. (e-mail: Elke.Scheer@ phys.uni-karlsruhe.de).

\section{Cation effects in doped $\mathrm{La}_{2} \mathrm{CuO}_{4}$ superconductors}

\section{J. P. Attfield, A. L. Kharlanov ${ }^{\star} \&$ J. A. McAllister ${ }^{\star}$}

Interdisciplinary Research Centre in Superconductivity, University of Cambridge, Madingley Road, Cambridge CB3 OHE, UK, and Department of Chemistry, University of Cambridge, Lensfield Road, Cambridge CB2 1EW, UK

* These authors contributed equally to the work

The critical temperatures of $\left(\operatorname{Ln}_{1-x} \mathrm{M}_{x}\right)_{2} \mathrm{CuO}_{4}$ superconductors ${ }^{1}$, in which $\mathrm{Ln}^{3+}$ (La and other lanthanides) and $\mathrm{M}^{2+}$ (Ca, Sr, Ba) cations are randomly distributed amongst the 'type A' lattice sites, are known to depend on the doping level, $x$, and the mean A-site cation radius, $\left\langle r_{\mathrm{A}}\right\rangle$ (refs 2,3 ). Here we show, by studying series of compositions with the same doping level and $\left\langle r_{\mathrm{A}}\right\rangle$, that the critical temperature decreases linearly with increasing A-site disorder, as quantified by the variance in the distribution of A-site cation radii. From this, we are able to show that, in the absence of disorder, the critical temperature should increase quadratically with $\left\langle r_{\mathrm{A}}\right\rangle$ for superconductors containing a single $\mathrm{CuO}_{2}$ layer. Our results therefore show that the critical temperature is very sensitive to lattice strains, as has also been shown for the metal to insulator transition temperature in the magnetoresistive $\left(\mathrm{Ln}_{1-x} \mathrm{M}_{x}\right) \mathrm{MnO}_{3}$ perovskites ${ }^{4}$.
Study of a series of $\left(\operatorname{Ln}_{0.7} \mathrm{M}_{0.3}\right) \mathrm{MnO}_{3}$ perovskites in which the mean A-site cation radius $\left\langle r_{\mathrm{A}}\right\rangle$ was held constant has showed ${ }^{4}$ that the metal-insulator transition temperature $T_{\mathrm{m}}$ decreases linearly with the statistical variance $\sigma^{2}$ in the distribution of A-site radii ${ }^{5}$, $\sigma^{2}=\left\langle r_{\mathrm{A}}^{2}\right\rangle-\left\langle r_{\mathrm{A}}\right\rangle^{2}$. The hole-doped $\left(\operatorname{Ln}_{1-x} \mathrm{M}_{x}\right)_{2} \mathrm{CuO}_{4}$ superconductors are similar to the above perovskites in that the doping level and lattice effects are controlled by the $\mathrm{Ln}^{3+}$ and $\mathrm{M}^{2+}$ cations at the A-sites, which in this case are adjacent to two-dimensional $\mathrm{CuO}_{2}$ perovskite layers. Many compositions with the 'optimum' doping level $x=0.075$, which maximizes the superconducting critical temperature $T_{\mathrm{c}}$ in the $\left(\mathrm{La}_{1-x} \mathrm{Sr}_{x}\right)_{2} \mathrm{CuO}_{4}$ system ${ }^{6}$, have been reported, and the variation of $T_{\mathrm{c}}$ with $\left\langle r_{\mathrm{A}}\right\rangle$ for series of $\left(\mathrm{La}_{0.925} \mathrm{Sr}_{0.075-x} \mathrm{Ba}_{x}\right)_{2} \mathrm{CuO}_{4}$ (ref. 7) and $\left(\mathrm{La}_{0.925-x} \mathrm{Ln}_{x} \mathrm{Sr}_{0.075}\right)_{2} \mathrm{CuO}_{4}(\mathrm{Ln}=\mathrm{Pr}, \mathrm{Nd}, \mathrm{Sm}, \mathrm{Eu})^{3,8}$ compositions are shown in Fig. 1. Systematic changes with a maximum $T_{\mathrm{c}}$ at $\left\langle r_{\mathrm{A}}\right\rangle=1.22 \AA$ are observed, but $T_{\mathrm{c}}$ is not uniquely determined by $\left\langle r_{\mathrm{A}}\right\rangle$ at the given doping level.

To determine whether $T_{c}$ is sensitive to A-site disorder, we have prepared three series of $\left(\mathrm{La}_{0.925-f} \mathrm{Nd}_{f} \mathrm{Sr}_{0.075-g-h} \mathrm{Ca}_{g} \mathrm{Ba}_{h}\right)_{2} \mathrm{CuO}_{4}$ compositions with the fixed $x=0.075$ doping level (Table 1). Values of $\left\langle r_{\mathrm{A}}\right\rangle$ and $\sigma^{2}$ were calculated from reported ionic radii for nine-coordination ${ }^{5}$. Initially, 15 compositions were prepared with $\left\langle r_{\mathrm{A}}\right\rangle=1.223 \AA$, the value for $\left(\mathrm{La}_{0.925} \mathrm{Sr}_{0.075}\right){ }_{2} \mathrm{CuO}_{4}$ (the $f=g=h=0$ composition). The A-cation size disorder varies between

\section{Table 1 Properties of three $\mathrm{A}_{2} \mathrm{CuO}_{4}$ series with fixed mean A-site radius}

A-site composition $\stackrel{\sigma^{2}}{\left(\AA^{2}\right)}$

$\left\langle r_{A}\right\rangle=1.212 \AA$ series

$\mathrm{La}_{0.899} \mathrm{Nd}_{0.026} \mathrm{Ca}_{0.075}$

$\mathrm{La}_{0.868} \mathrm{Nd}_{0.057} \mathrm{Ca}_{0.069} \mathrm{Ba}_{0.006}$

$\mathrm{La}_{0.825} \mathrm{Nd}_{0.100} \mathrm{Ca}_{0.061} \mathrm{Ba}_{0.014}$

$\mathrm{La}_{0.781} \mathrm{Nd}_{0.144} \mathrm{Ca}_{0.053} \mathrm{Ba}_{0.022}$

0.0000

0.0006

0.0012

0.0019

31.6

A $=1.223 \AA$ series

$\mathrm{La}_{0.925} \mathrm{Sr}_{0.075}$

0.0006

0.0009

$\mathrm{a}_{0.925} \mathrm{Sr}_{0.060} \mathrm{Ca}_{0.008} \mathrm{Ba}_{0.007}$

$\mathrm{La}_{0.925} \mathrm{Sr}_{0.045} \mathrm{Ca}_{0.017} \mathrm{Ba}_{0.013}$

$\mathrm{La}_{0.925} \mathrm{Sr}_{0.030} \mathrm{Ca}_{0.025} \mathrm{Ba}_{0.020}$

$\mathrm{La}_{0.925} \mathrm{Sr}_{0.015} \mathrm{Ca}_{0.033} \mathrm{Ba}_{0.027}$

$\mathrm{La}_{0.925} \mathrm{Sr}_{0.008} \mathrm{Ca}_{0.037} \mathrm{Ba}_{0.030}$

$\mathrm{La}_{0.925} \mathrm{Ca}_{0.041} \mathrm{Ba}_{0.034}$

$\mathrm{La}_{0.900} \mathrm{Nd}_{0.025} \mathrm{Ca}_{0.037} \mathrm{Ba}_{0.038}$

$\mathrm{La}_{0.875} \mathrm{Nd}_{0.050} \mathrm{Ca}_{0.032} \mathrm{Ba}_{0.043}$

$\mathrm{La}_{0.850} \mathrm{Nd}_{0.075} \mathrm{Ca}_{0.028} \mathrm{Ba}_{0.047}$

$\mathrm{La}_{0.825} \mathrm{Nd}_{0.100} \mathrm{Ca}_{0.023} \mathrm{Ba}_{0.052}$

$\mathrm{La}_{0.800} \mathrm{Nd}_{0.125} \mathrm{Ca}_{0.019} \mathrm{Ba}_{0.056}$

$\mathrm{La}_{0.775} \mathrm{Nd}_{0.150} \mathrm{Ca}_{0.014} \mathrm{Ba}_{0.061}$

$\mathrm{La}_{0.750} \mathrm{Nd}_{0.175} \mathrm{Ca}_{0.009} \mathrm{Ba}_{0.066}$

$\mathrm{La}_{0.725} \mathrm{Nd}_{0.200} \mathrm{Ca}_{0.005} \mathrm{Ba}_{0.070}$

$\left\langle r_{\mathrm{A}}\right\rangle=1.232 \AA$ series

$\mathrm{La}_{0.925} \mathrm{Sr}_{0.019} \mathrm{Ba}_{0.056}$

$\mathrm{La}_{0.921} \mathrm{Nd}_{0.004} \mathrm{Sr}_{0.018} \mathrm{Ba}_{0.057}$

$\mathrm{La}_{0.906} \mathrm{Nd}_{0.019} \mathrm{Sr}_{0.013} \mathrm{Ba}_{0.062}$

$\mathrm{La}_{0.888} \mathrm{Nd}_{0.037} \mathrm{Sr}_{0.007} \mathrm{Ba}_{0.068}$

$\mathrm{La}_{0.868} \mathrm{Nd}_{0.058} \mathrm{Ba}_{0.075}$

0.0012

0.0015

0.0019

0.0020

0.0022

0.0025

0.0029

0.0033

0.0036

0.0040

0.0043

0.0047

0.0050

Here $\sigma^{2}$ is the cation size variance, and $\left\langle r_{A}\right\rangle$ is the fixed mean A-site radius. A dash in the rightmost column indicates that superconductivity was not detected.

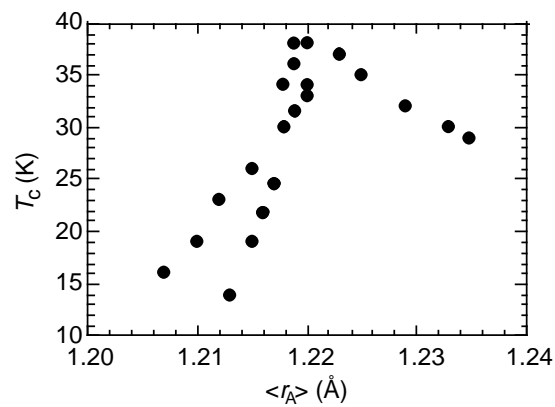

Figure $1 \mathrm{Plot}$ of $T_{\mathrm{C}}$ for previously reported $\left(\mathrm{Ln}_{0.925} \mathrm{M}_{0.075}\right)_{2} \mathrm{CuO}_{4}$ materials $\mathrm{S}^{3,7,8}$ against average A-cation radius $\left\langle r_{A}\right\rangle$. 